\title{
Sensor for Blood Outflow in Intravenous Intervention Detection
}

\author{
Lubos Sobota, Marek Penhaker, Jan Kubicek* \\ VSB-Technical University of Ostrava, FEECS, K450 17. listopadu 15, 708 33, Ostrava-Poruba, Czech Republic
}

\begin{abstract}
This paper deals with an issue, resulting in exceptional cases in intraaortic balloon counter pulsation. After analyzing the problem and find a worthy solution for detecting small concentrations of blood mixed with water content in the transparent tube, the first two chapters, optoelectronic detection method chosen. This principle is in the third part, which can be considered as the know-how of the entire thesis, 7 complex experimental measurements verified the accuracy. In this section, selected the best optoelectronic couple, the ideal setting and the algorithm chosen to evaluate the content of the tube. The final chapter is a self-designed device, called INKE1, which is able to repeatedly identify the contents in the tubing from the air, water and up to $0.1 \%$ of blood in the water.
\end{abstract}

Keywords: sensor; blood flow; intravenous intervention detection; counter pulsation.

\section{Introduction}

The Intra-aortic balloon pump (IABP) is a mechanical device that increases myocardial oxygen perfusion while at the same time increasing cardiac output. Increasing cardiac output increases coronary blood flow and therefore myocardial oxygen delivery. It consists of a cylindrical polyethylene balloon and contra pulsates. That is, it actively deflates in systole, increasing forward blood flow by reducing after load. It actively inflates in diastole, increasing blood flow to the coronary arteries. These actions combine to decrease myocardial oxygen demand and increase myocardial oxygen supply. A computer-controlled mechanism inflates the balloon with helium from a cylinder during diastole, usually linked to either an electrocardiogram (ECG) or a pressure transducer at the distal tip of the catheter. Helium is used because its low viscosity allows it to travel quickly through the long connecting tubes, and has a lower risk of causing an embolism should the balloon rupture. $[1,2,3,4,5]$

\subsection{Contra pulsation equipment}

There are two producers of the IABP in the Czech market: Arrow and Maquet, both american companies. Maquet produces the device CS300 and Arrow produces the device AutoCat 2 WAVE. Both devices have got its advantages. We are more acquainted with the device AutoCat 2 WAVE. There is a very slight possibility during the usage of this device that an undesirable appearance may occur: the pump might absorb a slight amount of blood, it is then blend in water, which circulates in the hose. $[9,10]$ The blood condensates and makes a haze, it is transferred in the hose to the IABP itself and might cause an error. It is desirable to eliminate this effect. $[7,8]$

\section{Methods}

The paper discusses first different measurement methods which could be used for the construction of detection device. One of the conditions which a detection 
device should comply with is that the measurement should be non- invasive, i.e. the device should not interfere in the IABP itself. Under these conditions there are 3 possible ways for measuring: capacitive, magnetic, optoelectronic. The first two means were denied because of a low possibility to distinguish the two substances (water and blood) in the hose. The most convenient base for the measurement is the optoelectronic one.

\subsection{The concept of the blood detection device}

The main part of the paper presents the development and construction of the blood detection device and experiments. Two experimental modules were constructed for the purposes of the experiments. The goal of the experiments was to find an ideal solution how to distinguish the content of the substances in a hose, i.e. to detect air, water and blood condensation.

The chosen method for the measurement is optoelectronic, it demands two components: a transmitter and a transceiver. The main criteria are wavelength, radiation angle, shape and intensity of the radiation. The only parameter which can directly influence the work in the experiments is the intensity of radiation. Since LED is part of the non-linear, as a reference while recording data of measured experimental measurements serve stream throughout the LED diode.

\section{Design and Realization of Sensor}

There were realized two measuring modules. The first one was used for the first four measurements, where the most convenient optoelectronic pair should be found. The second one was used for the next three measurements to find an ideal setting for the selected optoelectronic pair. There were used air, water and human blood for the experiments.

\subsection{Sensor prototype 1}

The next scheme presents the integration of the measuring module 1.

There were four measuring on this device, together 2250 values were recorded. The measuring followed each other analogically. During the gradual selection of the optoelectronic pairs, where there were 12 pairs in the measuring 1 and later modified, appeared as the most ideal optoelectronic pair for the blood and water detection in the contact of a hose, appeared a pair with three colored LED diode. Diode has a type designation LED $5 \mathrm{~mm}$ RGB-CC-4000/40 operating at wavelengths of $525 \mathrm{~nm}$ (detection of

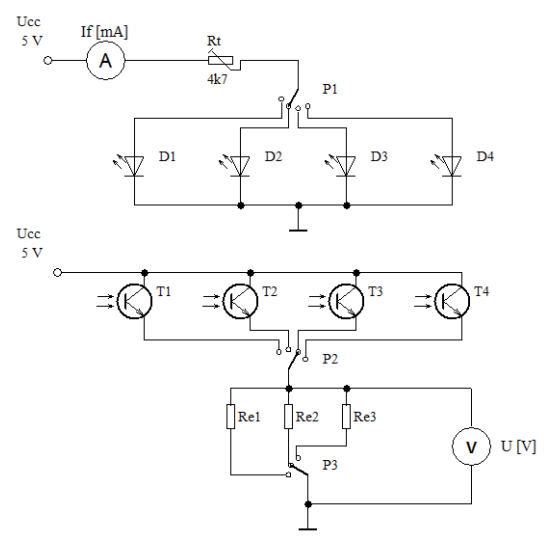

Fig. 1: Measurement module 1 for prototype alcohol sensor.

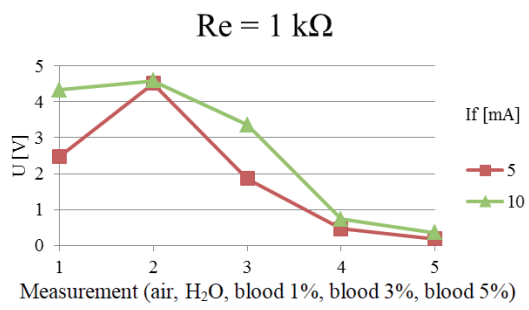

Fig. 2: Measurement ob blood in vater at wavelength $525 \mathrm{~nm}$.

blood) and $635 \mathrm{~nm}$ (detection of water). Electro optical transistor has a type designation, with a large TEPT5600 spectral sensitivity of the wavelengths.

Individual charts were compiled for the sensitivity of the phototransistor in the opto pair. The perimeter of the sensitivity is represented by the resistance $R e$, it is essentially the measured voltage drop in the resistance plotted on the $y$-axis The $X$ axis are individual measurements, which are always changing content in the tube. The same graph can also be found at different values of $I F$, ie the brightness LEDs.

\subsection{Sensor Prototype 2}

The second series of measurements (measurements 5,6 and 7) was carried out on by the author constructed measuring module 2. The aim of the measurements is finding of the optimal petting of the optoelectronic pair, which was chosen previously.

The next scheme presents the integration of the measuring module 2. Graph of the measured values of electro-pairs have the same wavelength of 525 $\mathrm{nm}$. As previous type of measurement. The graphs show the data measured in two tri-color LEDs. The charts were searched as far as the slope between 
the second and third measurement, ensuring the greatest sensitivity at $0.1 \%$ contained blood in distilled water.

There were three comprehensive measurements on this module. The first measurement (measurement 5) had the objective to state an appropriate algorithm, which will be in practical realization used for the detection of the hose content, i.e. unambiguous and repeatable detect air, water and blood-water solution.

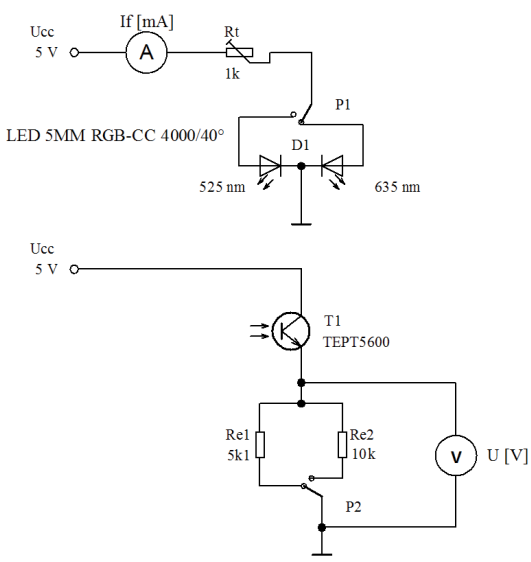

Fig. 3: Interconnection of measurement module 2 for sensor prototype.

The second measurement searched whether there is the analogy of the measure data between the first (air in the hose) and second (distilled water in the hose) measurement on in different hoses. It was appeared that there is no analogy.

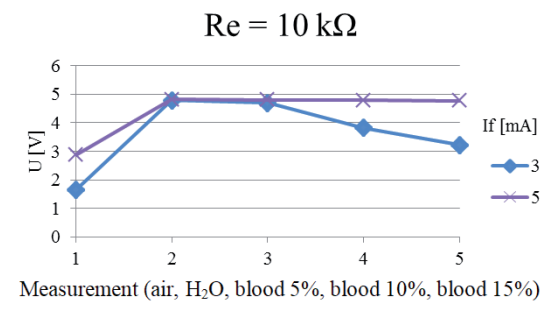

Fig. 4: Measurement ob blood in vater at wavelength $890 \mathrm{~nm}$.

From the measured data are the best results for optoelectronic pair operating at a wavelength of $890 \mathrm{~nm}$. Good sensitivity of the results reached and optoelectronic pairs with tri-color LED. With regard to measurement 2, but where optoelectronic couple do not have enough sensitivity to their second wavelength of $525 \mathrm{~nm}$, is excluded from the selection. In another measure to compare these pairs, with slight modifications, and decides for itself whether the detection of blood in the tubing used a three-color LED or two LEDs.

\section{Testing}

The last measurement reflected the requirements for the practical realization. The requirement was to reduce the supply voltage measuring optoelectronic transistor from $5 \mathrm{~V}$ to $2.8 \mathrm{~V}$. It was proven that there is no deterioration in the sensitivity of the optoelectronic transistor. The second objective was to find the most optimal petting of the optoelectronic pair, which would enable repeatable detection of substances, occurred in the hose.

Tab. 1: Prototype testing of quantity of blood leaking.

\begin{tabular}{|c|c|c|c|c|}
\hline \multicolumn{5}{|c|}{ Daily light } \\
\hline \multirow[t]{2}{*}{$\mathrm{n}$. } & \multicolumn{4}{|c|}{ Sample in hose } \\
\hline & air & water & $0,1 \%$ & $0,5 \%$ \\
\hline 1 & K & $P$ & A & \\
\hline 2 & K & $P$ & A & \\
\hline 3 & K & $P$ & $P$ & A \\
\hline 4 & P & A & & \\
\hline 5 & K & P & P & A \\
\hline 6 & K & P & A & \\
\hline 7 & P & P & A & \\
\hline 8 & K & P & P & A \\
\hline 9 & K & $P$ & A & \\
\hline 10 & K & $P$ & $P$ & A \\
\hline 11 & K & P & P & A \\
\hline
\end{tabular}

Results from the first test are reported in table 1. The table shows when and what state it was an INKE1 detected. K, P, and are the initial letters of each state they represent, ie Peace, P Warning, and Alarm. Cells filled with the color to green, showing good performance. Red filled cell shows the state erroneously reported. Cell filled with light orange color indicates a neutral evaluation of test performed. Line number represents number of tube test was performed.

\section{Results}

The blood detection in the hose is based on the principle of illuminating two wave lengths of the values of $525 \mathrm{~nm}$ and $635 \mathrm{~nm}$. These two wavelengths are contained in one component, the 
LED diode of the type-designation LED $5 \mathrm{~mm} \mathrm{RGB-}$ CC- $4000 / 40^{\circ}$.

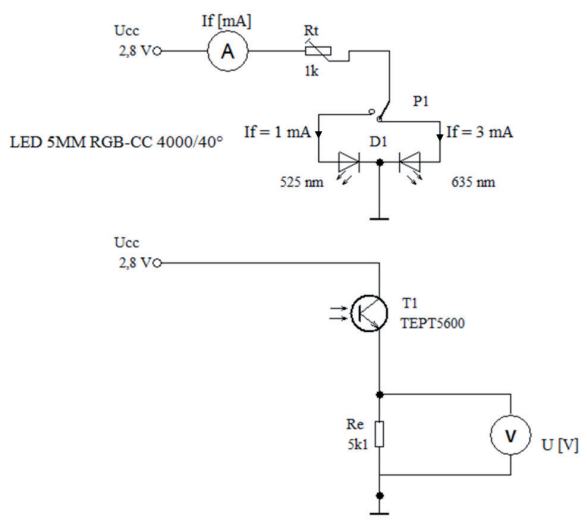

Fig. 5: Electrical diagram of opt electrical senor for blood and water detection.

Knot of photons emitted by this component goes through the content of the hose, where a refraction or absorption (by the material contented in the hose) occurs and it subsequently strikes on the photosensitive surface of the optoelectronic transistor. The optoelectronic transistor either opens (this causes more shrinkage on the resistance Re), or closes (this causes less shrinkage of voltage on the resistance $\mathrm{Re}$ ). This loss of the voltage on the resistance $R e$ is measured by voltmeter. A change of stream If measured by ammeter is set up by trimmer Rt. This value may give some analogy to the intensity of the luminous intensity of the components of LEDs, signed in the circuit D1, by the chosen wavelength with the switch P1. The optoelectronic transistor must be completely isolated from surrounding interfering light sources during the detection. In the subsequent construction also the distance between the boxes of the optoelectronic pair must be taken into account, it is the distance of $20 \mathrm{~mm}$.

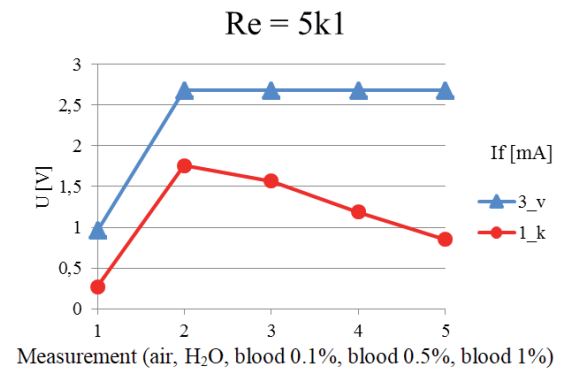

Fig. 6: Leak detection method of evaluating blood.
One of the objectives of the paper is to be able repeatedly distinguish the content of the hose from clean water and water blended with a small amount of blood (c. 0,5\%). Bud conditions in a hose are not permanent, there is gas helium and liquid water. This makes difficult the identification itself. Therefore two wavelengths $525 \mathrm{~nm}$ a $635 \mathrm{~nm}$ were chosen. The wavelength $525 \mathrm{~nm}$ is used to detect blood in a hose (because in this part of spectrum blood has got the best absorption according to the measured data). The wave length $635 \mathrm{~nm}$ is used to detect liquid- water in a hose, regardless of the content (in this case blood).
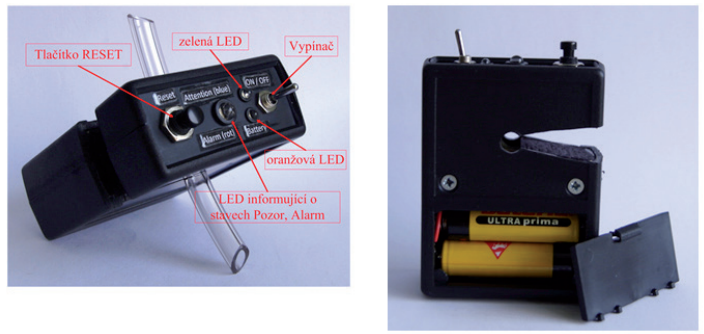

Fig. 7: Final prototype of blood leak detection.

The most optimal curve in the graph constructed from the measured date on the wave lengths 525 $\mathrm{nm}$ and $635 \mathrm{~nm}$ and different values If and Re was found. It was such curve, that both bisectors (bisector on $635 \mathrm{~nm}$ and bisector on $525 \mathrm{~nm}$ ) between measuring 1 and 2 were parallel. For those two curves the difference grows with the rise of the blood content in the solution, as reflected in the following graph. This difference is a determining figure for the subsequent detection of blood contained in water.

\section{Conclusions}

The paper deals with leak detection in blood during therapy and contra pulsation means assessing the quantity of leaking blood. There is described optoelectronic technique for detecting blood leakage. This method was subsequently tested experimentally seven measurements. The individual experiments were produced by two measurement.

To detect blood and water content in the tube, taking into account the characteristics of the actual detection and subsequent practical viability were choose Tri-color LED. Diodes operating at 
wavelengths of $525 \mathrm{~nm}$ (detection of blood), and $635 \mathrm{~nm}$ (detection of water). Phototransistor to detect the absorption of the type designation TEPT5600, possessing the high spectral sensitivity to those wavelengths. LED can work even for the third wavelength, but that would not apply and it is unnecessary to its value. The core of the evaluation of the sensor is ATtiny 13 microcontroller, manufactured by Atmel. The total current draw blood detector had a maximum value of $5.4 \mathrm{~mA}$, which gives a presumption of long-time active reliable operation. Made sensor to detect the complex in two subsequent tests successfully tested.

\section{Acknowledgments}

The work and the contributions were supported by the project SV4506631/2101 'Biomedicínské inženýrské systémy XII'.

\section{References and Notes}

[1] Taparia, N., Platten, K.C., Anderson, K.B., Sniadecki, N.J. A microfluidic approach for hemoglobin detection in whole blood (2017) AIP Advances, 7 (10), art. no. 105102.

[2] Liou, J.-C., Lin, W.-C., Kong, Y.-Y., Fang, K.-W. Investigated blood flow measurement of lights with different wavelengths system (2017) 2017 IEEE International Conference on Consumer Electronics - Taiwan, ICCE-TW 2017, art. no. 7991014, pp. 99-100.

[3] Ramírez-Miquet, E.E., Perchoux, J., Da Costa Moreira, R., Luna-Arriaga, A., Zhaoa, Y., Tronche, C., Sotolongo-Costa, O. Optical feedback interferometry: From basics to applications of laser flowmetry (2017) Revista Cubana de Fisica, 34 (1), pp. 48-57.

[4] Li, G., Lee, D.-W. An advanced selective liquid-metal plating technique for stretchable biosensor applications (2017) Lab on a Chip, 17 (20), pp. 3415-3421.

[5] Luo, C.-H., Su, C.-J., Huang, T.-Y., Chung, C.-Y. Non-invasive holistic health measurements using pulse diagnosis: I. Validation by three-dimensional pulse mapping (2016) European Journal of Integrative Medicine, 8 (6), pp. 921-925.

[6] Ullah, H., Ahmad, E., Hussain, F. Optical coherence tomography as glucose sensor in blood (2016) Advanced Structured Materials, 79, pp. 397-428

[7] Fine, I., Kaminsky, A.V., Shenkman, L. A new sensor for stress measurement based on blood flow fluctuations (2016) Progress in Biomedical Optics and Imaging - Proceedings of SPIE, 9707, art. no. 970705.

[8] Li, N., Jia, L., Zhang, P. Detection and volume estimation of bubbles in blood circuit of hemodialysis by morphological image processing (2015) Proceedings of the 2015 7th IEEE
International Conference on Cybernetics and Intelligent Systems, CIS 2015 and Robotics, Automation and Mechatronics, RAM 2015, art. no. 7274625, pp. 228-231.

[9] Siegel, G., Meyer-Rath, G., Ermilov, E., Rodríguez, M., Malmsten, M., Claesson, P., Saunders, R., Hetzer, R., Lindman, B. Flow sensing in the cardiovascular system (2015) Colloids and Surfaces A: Physicochemical and Engineering Aspects, 480, pp. 318-327.

[10] Aragón, A.M., Hernando-Rydings, M., Hernando, A., Marín, P. Liquid pressure wireless sensor based on magnetostrictive microwires for applications in cardiovascular localized diagnostic (2015) AIP Advances, 5 (8), art. no. 087132.

\section{Biographical notes}

Lubos Sobota: is a student of the Biomedical Engineering on the VŠB Technical University of Ostrava. He graduated on the master studies in 2011. His research includes biomedical devices and sensors.

Marek Penhaker: received his M.Sc. from Measurement and Control in 1996. He finished Ph.D in 2000 at VSB-TU Ostrava.from 2016 he is associate professor on CVUT Prague at Biomedical Engineering branch. His research interests include biomedical engineering, especially medical devices and bio-telemetry.

Jan Kubicek: was born in Ostrava, The Czech Republic. He received his M.Sc. from The Biomedical engineering in 2012. His research interests include applied image and signal processing in medicine and statistical analysis of biomedical data. 


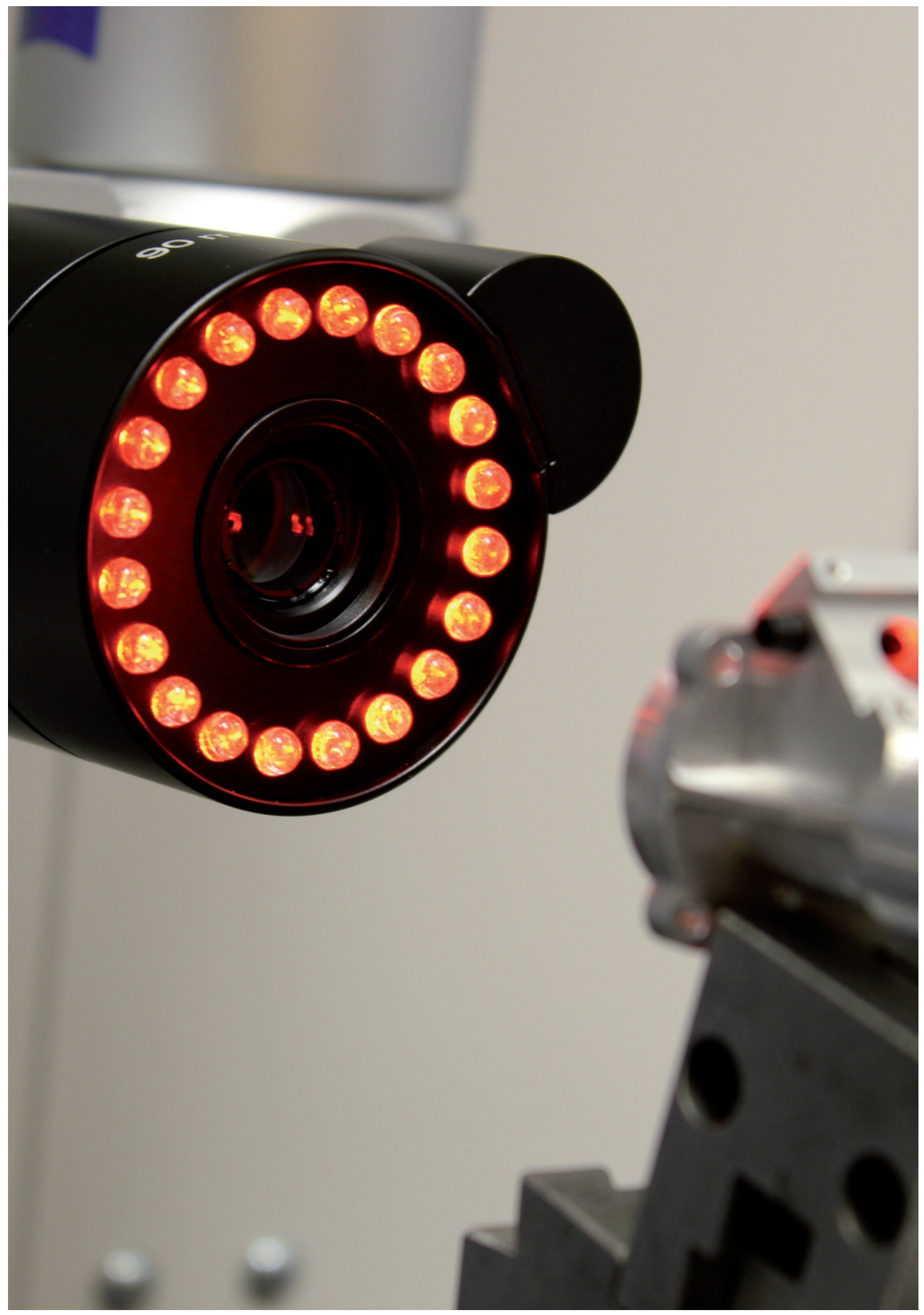

